\title{
Leaky Coaxial Cable with Enhanced Radiation Performance for Indoor Communication Systems
}

\author{
Zeeshan Siddiqui ${ }^{1}$, Marko Sonkki ${ }^{1}$, Marko Tuhkala ${ }^{2}$, Sami Myllymäki ${ }^{2}$ \\ ${ }^{1}$ Centre for Wireless Communications (CWC), University of Oulu, Oulu, Finland, zeeshan.siddiqui@oulu.fi \\ ${ }^{2}$ Microelectronics Research Unit, University of Oulu, Oulu, Finland
}

\begin{abstract}
In this paper, a novel method to selectively enhance the radiation performance of coupled mode leaky coaxial cable (LCX) is proposed. The LCX's jacket is printed by periodically spaced metallic stripes, which partially converts the bounded monofilar mode into a radiating mode. This adds to the leaked bifilar mode, enhancing the radiation performance of the leaky cable. It is shown that the coupling loss can be significantly decreased by the addition of periodic metallic stripes over the jacket. The radio coverage, at the desired locations of an indoor environment can be enhanced by adding the metallic stripes over the installed leaky coaxial cable.
\end{abstract}

Index Terms-coupling loss, distributed antenna system, indoor wireless systems, leaky coaxial feeder.

\section{INTRODUCTION}

In recent years, a growing academic and commercial interest is found in exploring new applications of the leaky coaxial cable (LCX). Conventionally, it is used in mines and tunnels as its radiation properties very well match with the coverage requirements of a uniform, long and narrow area also termed as linear-cell environment [1]. The LCX dual nature, that is simultaneous guiding and radiation of electromagnetic wave, and simpler installation makes it an attractive choice for indoor coverage. The current research and development directions are focusing on higher frequencies and wider bandwidths with applications ranging from sensors to wireless LAN access systems [2]. It has been demonstrated that the LCX outperformed the conventional discrete antennas in terms of covering the blind zones and shadowed zones in an indoor propagation environment [3]. However, in the complex indoor propagation environment, for example large buildings having offices, corridors and halls, the LCX alone cannot be a viable solution. The uniform radiation nature of the LCX offers no flexibility for the coverage requirements of such spatially varying environments [4].

In an earlier communication, the authors proposed to load the slots of a couple mode cable with a copper etched dielectric stripe to enhance the radiation performance of the cable [5]. In this paper a modification related to the previous work is presented to selectively increase the radiation efficiency of an LCX by printing its jacket with periodically spaced metallic stripes. The presented solution is cost effective and simpler to fabricate. However, there is a trade-off, without an additional dielectric stripe, as there was in the previous work; the only available design parameter is the metallic patch dimensions.

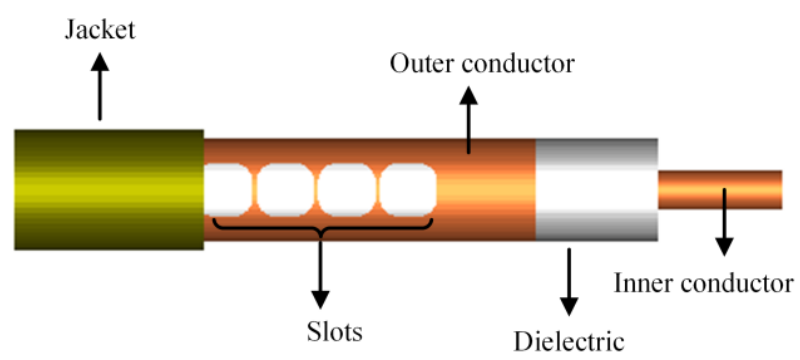

Fig. 1 Coupled mode leaky coaxial cable (LCX)

This limits the design freedom especially at lower frequencies. The cable radiation performance is improved as the monofilar mode, similar to surface wave is partially converted to propagating mode because of modulated surface reactance, as shown later in this paper. It adds to the existing leaked bifilar mode increasing the total radiation from the leaky cable. The cable can be printed on the selective locations according to coverage requirements. The focused frequency band is around 5 $\mathrm{GHz}$, which supports wireless LAN communications under IEEE 802.11 at different places.

\section{THEORETICAL BACKGROUND}

\section{A. Leaky Coaxial Cable (LCX)}

Fig. 1 shows a coupled mode leaky coaxial cable LCX. Its electrical performance is very similar to a axially slotted cable as the distance between the slots is very small relative to wavelength. It differs from an ordinary coaxial cable as it has slots over the outer conductor which leak the energy as the wave propagate along its length. In this way, it acts simultaneously as a wave guiding and radiating structure. LCX are generally categorized based on their radiation mechanism in two types, coupled mode and radiating mode cables. It depends primarily on the spacing between the slots. This paper focuses on the coupled mode cable, which has either an axial slot or very closely spaced slots. It has been established that the couple mode LCX supports two distinct modes, named as monofilar and bifilar [6]. The bifilar or the coaxial mode propagates between the center and outer conductor of the coaxial cable while some of its energy leaks outside from the slots. The cable's radiation performance is generally dependent on this leakage. In contrast, the monofilar mode propagates over the outer side of the outer conductor, similar to a bounded surface wave. By the introduction of periodically spaced metallic stripes over the 
(a)

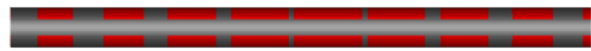

(b)

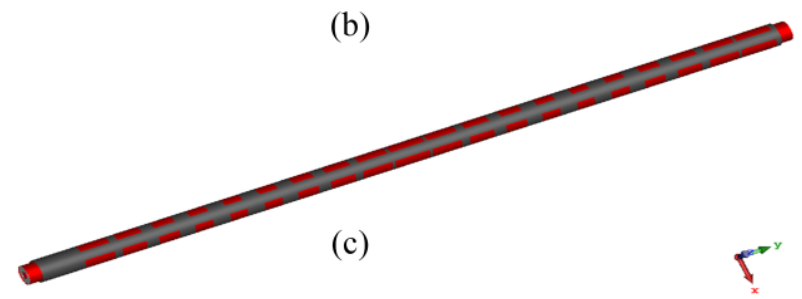

Fig. 2. CST MWS simulation model of a leaky coaxial cable with its jacket printed with periodically spaced metallic patches (a) zoomed section without jacket (b)zoomed section with printed metallic stripes over the jacket (b) Entire simulated model

jacket, the slow monofilar is partially converted to a radiating wave. This radiation is due to the phenomenon, when a surface wave is subjected to periodic impedance variation, it transforms in to leaky wave. In the following, theory describing this conversion is briefly presented.

\section{B. Sinusoidally Modulated Reactance Surface (SMRS)}

A plane surface whose modal surface impedance is sinusoidally modulated is termed as sinusoidally modulated reactance surface (SMRS). A. A. Oliner and A. Hessel theoretically investigated the propagation characteristics of the SMRS [7], and its relevance to surface wave antenna was demonstrated. It was proved that, periodic modulation of the surface reactance beyond a threshold period will cause single or multiple leaky wave to emanate from the bounded surface wave.

The surface impedance, $\eta$, of a SMRS in the $x y$-plane, with the direction of wave propagation assumed to be in $z$-direction, is given by following equation [7].

$$
\eta(z)=j \eta_{o} X\left[1+M \cos \left(\frac{2 \pi z}{a}\right)\right]
$$

Where $\eta_{o}$ is the free-space wave impedance, $X$ is the average surface reactance normalized by the free-space wave impedance, $M$ is the modulation factor and $a$ is the periodicity of modulation. The parameters $(X, M, a)$ are the design parameters of a periodically modulated reactance surface.

The discussed theory has been successfully applied to many surface wave antennas from microwave frequencies to $\mathrm{THz}$ range [8], [9] .

\section{Design And Simulation SetuP}

Initially, an LCX with very closely spaced small slots is designed and simulated using CST Microwave Studio. Small slots are equivalent to a long axial slot as the spacing between the slots is very small compared to the wavelength. Since the design is targeted to $5-6 \mathrm{GHz}$ frequency band, the simulated design followed the 0.5 -inch standard commercially available cable. The cut-off frequency for the cable is $9.8 \mathrm{GHz}$, beyond this frequency the higher order modes are also supported by the cable. The coaxial cable was designed with the inner and outer conductor diameters of $4.8 \mathrm{~mm}$ and $12.4 \mathrm{~mm}$, respectively. Cellular polyethylene with dielectric constant of 1.25 and loss

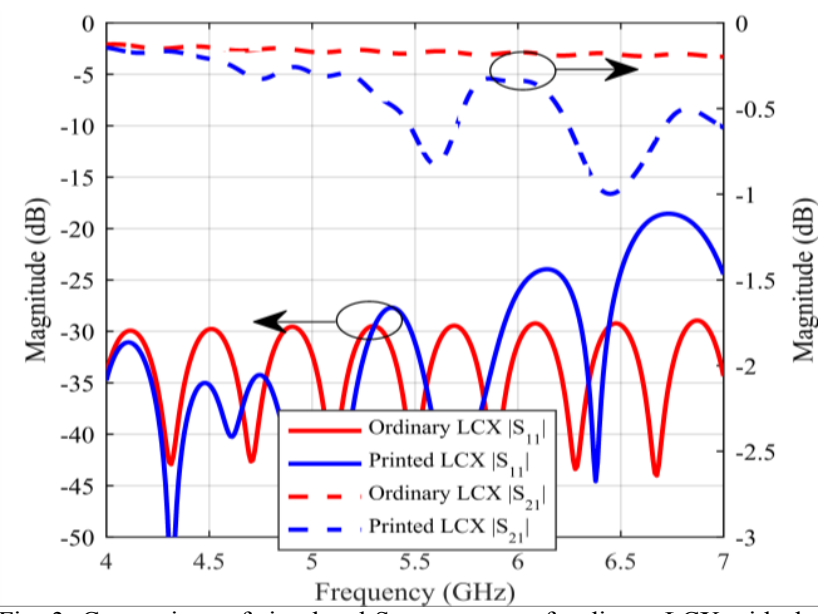

Fig. 3. Comparison of simulated S-parameters of ordinary LCX with the proposed LCX, having jacket printed with the metallic stripes.

tangent of 0.0002 is used as a dielectric material between the two conductors. Usually, the commercially available cables are with corrugated outer conductor. Here the outer conductor was kept plain to avoid complexity in the simulation model. The outer jacket dimensions also followed the off the shelf standard cable, where the thickness of the cable jacket is kept at $1 \mathrm{~mm}$ with the dielectric constant of 2.3. The slots were $7 \mathrm{~mm}$ in length and width with a spacing of $0.5 \mathrm{~mm}$ in between them. The selected frequency range was from $4 \mathrm{GHz}$ to $7 \mathrm{GHz}$, covering the target frequency bands for Wireless LAN communications. The simulated length of the cable was $0.44 \mathrm{~m}$, which was excited and terminated by waveguide ports. In addition, small sections of ordinary coaxial cable were placed closed to the ports in the simulation model.

In the second step, the metallic patches over the cable's jacket were simulated to enhance its radiation efficiency. Iterative simulations were performed to achieve the desired performance at the required frequency band. Theoretically the design is based on the fundamental parameters, surface reactance $X$, modulation factor $M$ and the periodicity $a$, which dictate the performance of an SMRS. In the presented work, full wave simulations were utilized to observe the effect of varying periodicity and modulation factor. The leakage rate is controlled by the modulation factor, $M$. The periodicity, $a$, was varied to tune the frequency band of operation. In the presented case, the objective was to partially convert the monofilar mode into leaky wave without completely changing the wave propagation and radiation mechanism of the LCX. As discussed earlier, the presented solution offers little flexibility in design process as the dielectric thickness and permittivity are fixed during the cable fabrication.

Here, two periods of the metallic patches were placed over the cable jacket. There are 10 patches in a period with periodically varying dimensions. The total period length was tuned to $160 \mathrm{~mm}$, by observing improvement in the radiation performance of the LCX. Two equi-dimensional patches are placed on either side of the slots as shown in the simulation model, Fig. 2. The additional resonances and a deteriorated impedance matching were observed with the patches covering the slot aperture. The patches were converted in to two along 


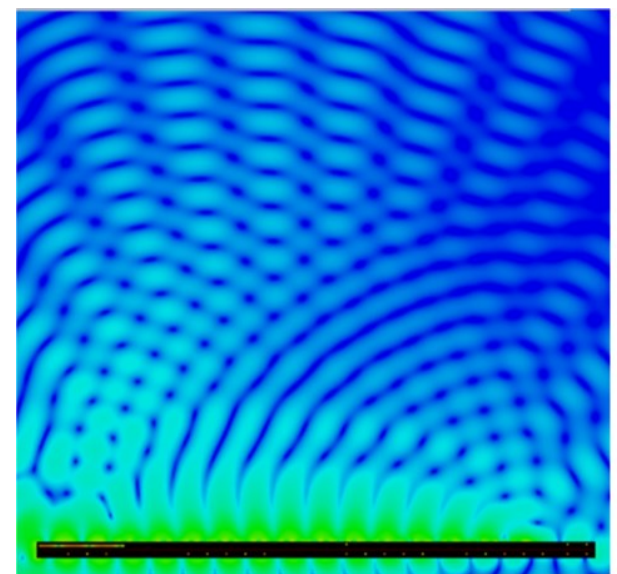

(a)

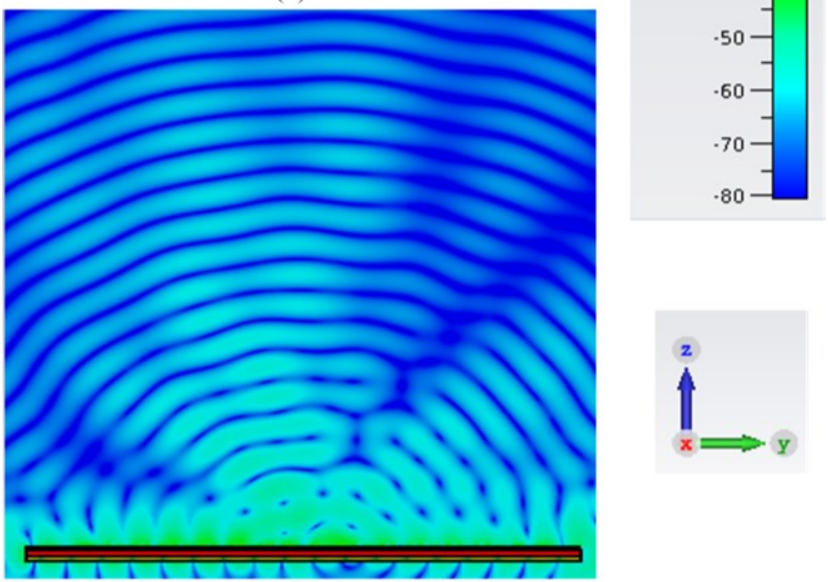

(b)

Fig. 4. 2D electric field plots at 5.5 GHz (a) Ordinary LCX (b) Proposed LCX.

sides of the slots, to clear the slot aperture. This caused a noticeable improvement in the matching. In the following simulated results are presented and discussed.

\section{RESUlts AND Discussion}

The LCX with printed metallic patches over its jacket is compared with an ordinary LCX to observe the differences and improvements in its electrical performance. The comparison is done in terms of S-parameters, 2D electric field plots, and coupling loss. Fig. 3 shows the S-parameters of the cables. The impedance matching remains same in the targeted $5 \mathrm{GHz}$ to 6 $\mathrm{GHz}$ band but slightly deteriorated at the higher frequencies due to the printed patches. The $\left|S_{21}\right|$ shows the power received at the terminated port, the lesser power received in case of the proposed cable shows that it has radiated more than the ordinary LCX if the limited copper losses are ignored. The 2D electric field strength plots at $5.5 \mathrm{GHz}$ mapped in Fig. 5 shows the different radiation mechanism of the two cables. The cables were vertically bisected from the top, showing the half-section in the yz-plane. The bifilar mode confined between the two conductors is strongest in both the cases. In the periphery, it is visible that the field strength was significantly higher in the loaded case. The visible difference between the two plots are the directional contours in the printed jacket case due to the transformation of surface wave in radiating waves.

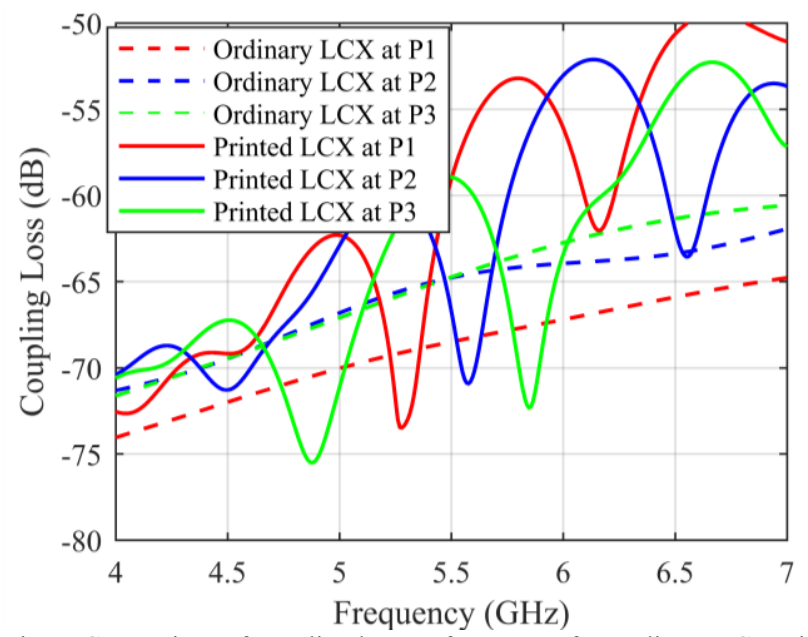

Fig. 5. Comparison of coupling loss performance of an ordinary LCX with the proposed LCX.

Coupling loss is an exclusive parameter to classify the performance of an LCX. Basically it is a ratio of received power

to transmitted power at a specific distance by standard dipole and defined by the following equation,

$$
\text { Coupling Loss }(\mathrm{dB})=-10 \log \left(\frac{P_{r}}{P_{t}}\right)
$$

where $P_{r}$ is the received power by the standard half-wavelength dipole antenna and $P_{t}$ is the input power. In simulations, the $P_{r}$ was calculated by the recorded electric field strength, $E$, utilizing the empirical relation, $0.13 \lambda^{2}|E| / 120 \pi$ [10], and $\mathrm{P}_{t}$ was set to 0.5 watt. Three electric field probes at different locations along the cable length at a vertical distance of $1 \mathrm{~m}$ were placed in the simulation model to record the electric field strength. Fig 5 depicts the decrement in coupling loss resulting from the placement of metallic patches over the jacket. The coupling loss is reduced by $5-10 \mathrm{~dB}$ in case of the proposed LCX. It is also observed that the loss is not uniform along the cable length and the operating frequencies. It may not be a significant issue in an indoor multipath environment as the coupling loss is measured and stated statistically. The far-field pattern remains similar to an ordinary LCX but the realized gain is increased by 6-7 dB in case of the proposed LCX.

\section{CONCLUSION}

In this paper, a solution to decrease the coupling loss of a coupled mode LCX by printing its jacket with periodically spaced metallic patches was presented. The proposed solution is particularly suited to high and wide frequency bands. The primary advantage of the design is its low cost and simple fabrication. Even after the installation of the LCX, these metallic patches in the form of sticker can be pasted at the locations where coverage is unsatisfactory. It can be concluded that placing periodically spaced metallic patches can complement the LCX performance at complex propagation environments especially at higher frequencies and wider bandwidths. 


\section{ACKNOWLEDGMENT}

The work was supported in part by the Finnish Funding Agency for Innovation, Tekes, under Smart Wireless Cable-Project and the Academy of Finland 6Genesis Flagship (grant no. 318927). We are grateful to our sponsors and the industrial collaborators, Prysmian Group, Elcoflex and Premix.

\section{REFERENCES}

[1] Yafei Hou et al, "Capacity Evaluation of MIMO Channel With One Leaky Coaxial Cable Used as Two Antennas Over Linear-Cell Environments," IEEE Transactions on Vehicular Techcnology, vol. 66, (6), pp. 4636-4646, Jun, 2017.

[2] Yiming Wu et al, "An Experimental Study of MIMO Performance Using Leaky Coaxial Cables in a Tunnel," Antenna and Wireless Propagation Letters, vol. 16, pp. 1663-1666, 2017.

[3] M. Nakamura, H. Takagi, K. Einaga, "Evaluation of a dual-band long leaky coaxial cable in the 2.4 and $5 \mathrm{GHz}$ frequency bands for wireless network access," IEEE Radio and Wireless Symposium, Jan 2009

[4] Jun Hong Wang, "Leaky coaxial cable with adjustable coupling loss for mobile communications in complex environments," IEEE Microwave and Wireless Components Letters, vol. 11, (8), pp. 346-348, 2001.

[5] Z. Siddiqui et al, "Sinusoidally Modulated Reactance Surface Loaded Leaky Coaxial Cable," European Conference on Antenna and Propagation, April 2019

[6] R. A. Hurd, "The modes of an axially slotted coaxial waveguide," Radio Science, vol. 14, (5), pp. 741, 1979.

[7] A. Oliner and A. Hessel, "Guided waves on sinusoidally-modulated reactance surfaces," IRE-Transactions on Antenna and Propagation, vol. 7 , (5), pp. 201-208, 1959.

[8] A. M. Patel and A. Grbic, "A Printed Leaky-Wave Antenna Based on a Sinusoidally-Modulated Reactance Surface," IEEE Transactions on Antennas and Propagation, vol. 59, (6), pp. 2087-2096, 2011.

[9] X. Bai et al, "Sinusoidally Modulated Leaky-Wave Antenna for MillimeterWave Application," IEEE Transactions on Antennas and Propagation, vol. 64, (3), pp. 849-855, Dec, 2016.

[10] Jun Hong Wang and K. K. Mei, "Theory and analysis of leaky coaxial cables with periodic slots," IEEE Transactions on Antennas and Propagation, vol. 49, (12), pp. 1723-1732, 2001 